\title{
Complete regression of primary melanoma associated with nevi involution under BRAF inhibitors: A case report and review of the literature
}

\author{
LAVINIA ELENA GRIGORE ${ }^{1,2}$, LOREDANA UNGUREANU ${ }^{1}$, NONA BEJINARIU ${ }^{3}$, CRINA SECEAC ${ }^{4}$, \\ ALINA VASILOVICI $^{1}$, SIMONA CORINA SENILA ${ }^{1}$, ELISABETA CANDREA ${ }^{1}$, \\ OANA FECHETE $^{1}$ and RODICA COSGAREA ${ }^{1}$ \\ ${ }^{1}$ Department of Dermatology, 'Iuliu Hațieganu' University of Medicine and Pharmacy, 400006 Cluj-Napoca; \\ ${ }^{2}$ Department of Dermatology, Municipal Clinical Hospital, 400139 Cluj-Napoca; ${ }^{3}$ Santomar Oncodiagnostic, \\ 400350 Cluj-Napoca; ${ }^{4}$ Medisprof Cancer Center, 400641 Cluj-Napoca, Romania
}

Received August 7, 2017; Accepted September 25, 2018

DOI: $10.3892 / \mathrm{ol} .2018 .9738$

\begin{abstract}
Melanoma is one of the most immunogenic tumors among human neoplasms, with numerous clinical observations of partial or completely regressed tumors. It is an aggressive tumor, with the greatest reported number of somatic mutations, BRAF mutation being the most common one. BRAF mutation is also present in a higher percentage in benign nevi. Complete regression of primary tumor and involution of nevi are, however, rare phenomenon in melanoma that can appear in relation with UV exposure, surgical trauma, target therapy in melanoma, pregnancy or host immune response to an evolving melanoma tumor. We present the case of a 58-year-old man with a completely regressed metastatic melanoma who developed a second melanoma with concomitant involution of papillomatous nevi under BRAF inhibitors treatment. In reviewed literature we have found 53 cases of completely regressed primary melanomas, neither of them reporting nevi involution. Complete regression of primary tumor can occur as an immune response to tumor progression. Nevi can involute under BRAF inhibitor therapy, but development of new malignant lesions under BRAF inhibitors is linked to BRAF wild-type. Documentation of primary tumor and dynamic changes in nevi highlight the need of a good clinical skin examination and increase the utility of baseline and sequential dermoscopy in melanoma.
\end{abstract}

Correspondence to: Dr Loredana Ungureanu, Department of Dermatology, 'Iuliu Hațieganu' University of Medicine and Pharmacy, 3-6 Clinicilor Street, 400006 Cluj-Napoca, Romania E-mail: danaszent@yahoo.com

Key words: regressed melanoma, nevi involution, dermoscopy, BRAF

\section{Introduction}

The regression of cutaneous pigmented lesions, benign or malignant, is a well-known phenomenon. The underlying mechanism is not completely understood, but factors like host immune system, UV radiation and trauma have been suggested. Melanoma is a multifactorial disease witch demonstrates a very complex interplay between identifiable risk factors such as UV exposure, phenotype and genotype (1-3). According to Bastian (4) melanoma risk increase with the presence of multiple enlarged acquired nevi. Melanomas can be epithelium-associated or not and can show different age distribution and different relationships to UV exposure.

Melanoma is the tumor with the highest number of reported somatic mutations across human cancer types (5). The most common one, that occurs in $\sim 50 \%$ of melanomas is BRAF mutation. His variant V600E is the most prevalent one and has been associated with younger age at diagnosis and location of primary tumor often on the trunk. BRAF V600E mutation is also present in $70 \%$ of benign nevi (6).

After the discovery of BRAF mutations, new targeted therapies like BRAF inhibitors have been introduced for the treatment of melanoma. Although well tolerated when used in clinical practice, they present some adverse events, cutaneous adverse events being the most common ones. Under BRAF therapy dynamic changes in melanocytic lesions like involution of nevi, changes in pigmentation and size and appearance of new melanomas have been described $(7,8)$.

Although partial regression is relatively frequent and occurs in $10-35 \%$ of cases, spontaneous complete regression with documented histology is very rare and occurs only in $0.22-0.27 \%$ of all melanoma cases (9).

\section{Case report}

A 58-year-old man presented in the Department of Surgical in 2014 with a $6 / 4.5 \mathrm{~cm}$ subcutaneous tumor on the left lateral chest wall of several months duration. Excision of the lesion revealed a nodular, solid, dermo-hypodermic proliferation 
A

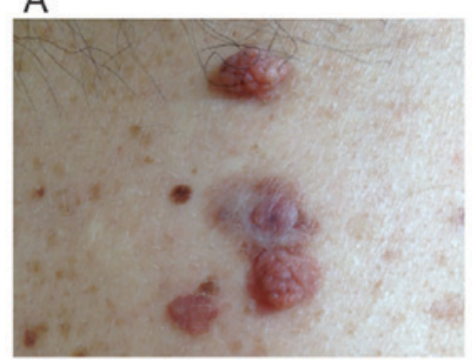

B

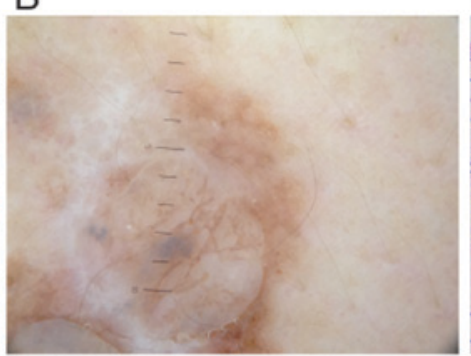

C

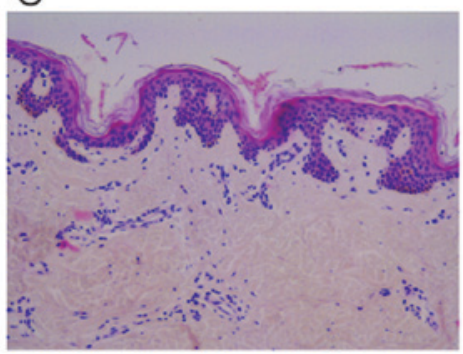

Figure 1. Clinical, dermoscopic and pathological presentation of a completely regressed melanoma. (A) Clinical presentation of the regressed melanoma showing a suspicious elevated lesion with grayish appearance. (B) Polarized dermoscopy of the regressed melanoma showing scar like depigmentation, pigmented globules and white transverse lighter bands. (C) Histologic analysis of the regressed melanoma showing flat rete ridges, lamellar fibrosis, increased vascularity, focal lymphocytic infiltrate with no melanocytic proliferation. Hematoxylin and eosin; magnification, x50.

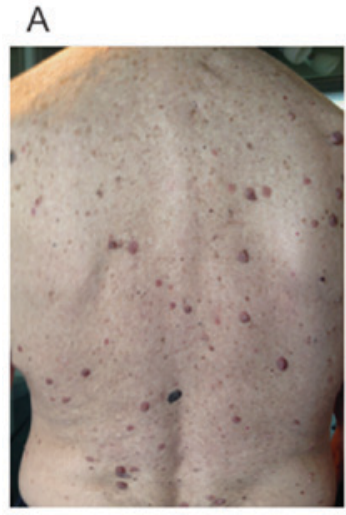

\section{B}

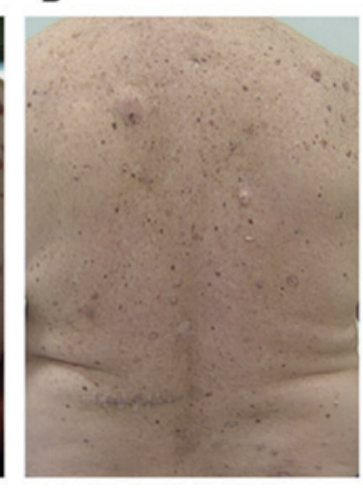

C

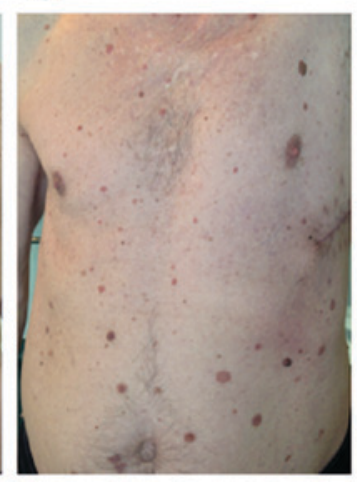

D

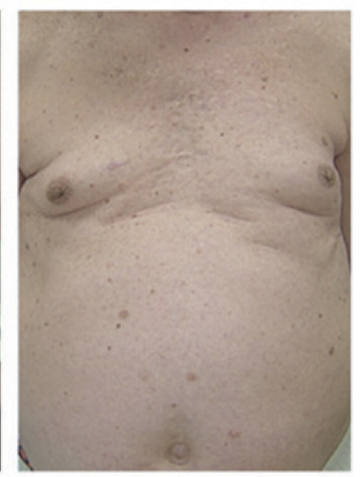

Figure 2. Clinical image of the patient before and after three years of BRAF therapy. (A) Baseline clinical evaluation of the back and (C) front before treatment. (B) Clinical evaluation of the back and (D) front after three years of BRAF inhibitors.

with large atypical round, polygonal cells, high number of mitosis (12/10 HPF) at pathological examination. It showed HMB45 and S100 positivity on immunohistochemical staining, concluding to an in-transit metastasis of unknown primary melanoma.

The clinical examination showed in the left lumbar region a suspicious elevated lesion, unknown to the patient, with grayish appearance (Fig. 1A) and multiple enlarged papillomatous nevi on the trunk (Fig. 2A). Polarized dermoscopy revealed scar like depigmentation, pigmented globules and white transverse lighter bands between two papillomatous lesions (Fig. 1B). The clinical and dermoscopic aspect of the lesion, the ipsilateral location indicated a regressed tumor, most likely the primary melanoma. He also presented leftsided axillary lymphadenopathy on clinical examination, confirmed by the CT scan. Surgery was performed on the suspicious lesion and axillary lymph nodes. Pathological examination of the regressed lesion showed flat rete ridges, lamellar fibrosis, increased vascularity, focal lymphocytic infiltrate with rare melanophages and no signs of melanocytic proliferation (Fig. 1C). Four of the 26 axillary lymph nodes removed showed tumoral cells with positivity for the HMB45 staining concluding to a lymph node melanoma metastasis. After surgery the patient showed rapid progression with detection of pleural metastasis on the CT scan. The patient was diagnosed with a stage IV melanoma with pleural metastasis and a completely regressed primary tumor. BRAF V600E mutation was detected in the subcutaneous lesion.
The patient started Vemurafenib treatment and showed partial response on the pulmonary CT scan after 3 months of therapy, but with numerous cutaneous adverse events: Multiple keratoachantomas, warts and changes in nevi color. One month later an atypical pigmented lesion (Fig. 3A and B) at the base of a papillomatous nevus that changed from baseline evaluation was excised. It turned out to be a $0.7-\mathrm{mm}$ Breslow index superficial spreading melanoma with less than 1 mitosis $/ \mathrm{mm}^{2}$, a brisk lymphocytic infiltrate and no regression present (Fig. 3C). In the same time involution of some papillomatous nevi was observed, by diminished size and lighter color. After other three months Vemurafenib treatment was stopped because of insurance issues and another BRAF inhibitor was started, Dabrafenib, which became available for BRAF mutated melanoma patients in Romania at that time.

After three years of BRAF inhibitor monotherapy all his papillomatous lesions involuted (Fig. 2B and D). The patient had excellent systemic response with stable disease and no cutaneous or systemic side effects from BRAF inhibitor therapy. He is still under surveillance.

The present case is interesting because the association of completely regressed melanoma with metastases, and development of a new lesion under BRAF treatment.

\section{Methods}

We performed a search on PubMed using the terms 'primary melanoma', 'spontaneous regression', 'complete regression' to 
A

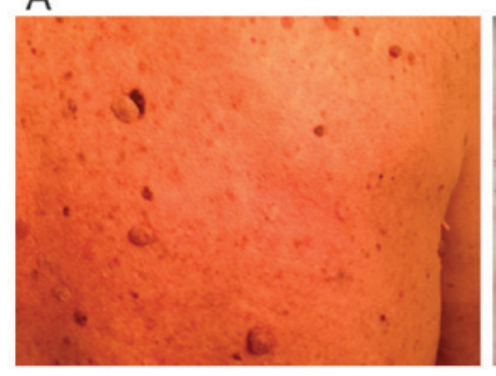

B

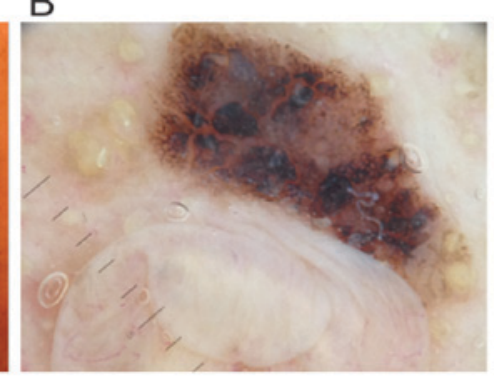

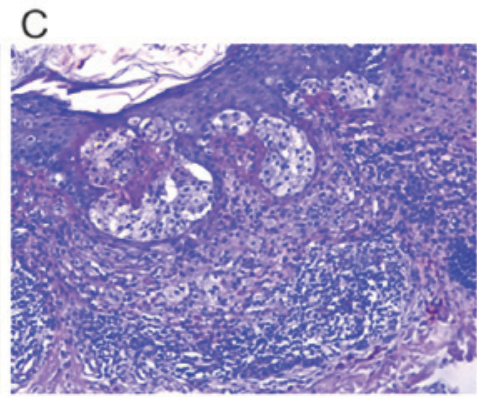

Figure 3. Clinical, dermoscopic and pathological presentation of the second melanoma under BRAF therapy. (A) Clinical presentation showing an atypical pigmented lesion at the base of a nonpigmented papillomatous nevus. (B) Polarized dermoscopy of the new melanoma showing flat eccentric pigmentation at the base of a nonpigmented papillomatous nevus. (C) Histologic presentation of the new melanoma showing nests of melanocites with variable size and shape, pagetoid spreading, large cells with abundant eosinophilic cytoplasm with prominent nuclei. Hematoxylin and eosin; magnification, x100.

identify similar cases, restricting the papers to those published in English or French. Additional articles using the reference lists of the identified papers were noted. We found 50 cases of fully regressed melanomas which developed metastasis and three cases of initially confirmed melanomas with complete regression after partial incisional biopsy. Two cases of completely regressed melanomas with no confirmed distant metastasis in spite of extensive work up for two year follow-up were excluded from this study $(10,11)$. Age, sex, location of the tumor and site of metastasis were noted for each case. We also recorded information about the diagnosis of the primary tumor, if it was retrospective or initial and pathologically confirmed. Dermoscopy of complete regressed melanomas was reported only in 10 cases (10-12).

We did not find in the literature any association between completely regressed melanomas and BRAF mutational status. Demographic and clinical information on the patients are summarized in Table I.

\section{Discussion}

Complete regression in melanoma is a rare event, being reported only in $0.22-0.27 \%$ of all melanoma cases (9). Clinical and pathological presentation of complete regressed melanomas have been extensively described through the literature. Dermoscopic evaluation of completely regressed melanoma is based only on a few case reports in the last 10 years (10-12). We did not find in the literature any association between completely regressed melanomas and BRAF mutational status. Nevi involution associated with melanoma was reported in the literature as a result of melanoma development followed by spontaneous regression of nevi or as a result of targeted therapy with BRAF inhibitors $(35,36)$.

Clinical presentation of completely regressed melanoma. Completely regressed melanoma usually presents as a hypopigmented macule with pink or scar-like appearance. In rare cases, they can also appear as either hyperpigmented macules, or with remnants of pigmentation as blue-gray discolorations (11). Our review showed that all melanomas were epithelium associated (4), with a male predominance representing $62.26 \%$ of all cases and a median age of 48.5 years. Eighty-three percent of cases presented in relation with low UV exposure in non-chronic sun damaged skin $(39,62 \%$ involving the trunk and $43.39 \%$ involving arms and legs) and only $16.98 \%$ were located on chronic sun damaged skin (cervical), in relation with high UV exposure. The diagnosis of regressed melanoma was consistent with criteria of Smith and Stehlin and was retrospective in all cases except one (14). Three pathologically confirmed melanomas (two superficial spreading and one nodular melanoma) completely regressed after incisional partial biopsy of primary tumor (32-34).

Dermoscopic presentation of completely regressed melanoma. There are no established dermoscopic criteria for the diagnosis of complete regressed melanomas. In the literature we found only 10 reported cases of completely regressed melanoma with pathological confirmation and dermoscopic evaluation. Seven cases are reported by Bories et al (12) who showed that the most frequent dermoscopic features present in all cases were scar-like depigmentation and pink background. Eighty-six percent of patients in his serie had linear irregular vessels as defined by Argenziano et al (37) and remnants of pigmentation on dermoscopic evaluation. Forty-three percent of patients in Bories et al (12) serie had 'peppering' as defined by Zalaudek et al (38), white transverse lighter bands in polarized dermoscopy and globular vessels. High et al (11) recorded just 'peppering' and dusty blue-gray coloration on dermoscopic evaluation, performed in only two of his 38 melanoma cases reported. Pozzobon et al (39) found that 'peppering' is a dermoscopic feature frequently associated with regression in BRAF mutated melanoma. They stated that this can be considered a morphological consequence of BRAF mutated melanoma biological behavior.

Our case presented on polarized dermoscopy: Scar-like depigmentation, pigmented globules and white transverse lighter bands, features associated with regression (Fig. 1B).

Scar-like depigmentation and white transverse lighter bands on a pink background were observed in our patient also at the level of some regressed papillomatous nevi.

Pathological analysis of completely regressed melanoma. To confirm the diagnosis of a completely regressed melanoma is often very difficult. The diagnostic approach is almost always retrospective, with primary lesion being detected in metastatic stage (11). There is only one reported case of complete primary regressed melanoma detected before metastatic stage (30). According to Massi and LeBoit (40) the important issue is 
Table I. Demographic and clinical information on the patients.

\begin{tabular}{|c|c|c|c|c|c|c|c|}
\hline Author (year) & Ref. & $\begin{array}{l}\text { No. of } \\
\text { patients }\end{array}$ & $\begin{array}{c}\text { Age } \\
\text { (years) }\end{array}$ & Sex & $\begin{array}{l}\text { Location of } \\
\text { primary tumor }\end{array}$ & Sites of metastasis & $\begin{array}{l}\text { Diagnosis of } \\
\text { primary tumor }\end{array}$ \\
\hline \multirow[t]{2}{*}{$\begin{array}{l}\text { Dasgupta et al, } \\
1963\end{array}$} & \multirow[t]{2}{*}{ (13) } & \multirow[t]{2}{*}{2} & 35 & M & Trunk & $\begin{array}{l}\text { Ipsilateral cervical and } \\
\text { axillary lymph nodes }\end{array}$ & $\begin{array}{l}\text { Retrospective/skin } \\
\text { examination/excisional biopsy }\end{array}$ \\
\hline & & & 49 & M & Trunk & $\begin{array}{l}\text { Ipsilateral axillary } \\
\text { lymph nodes }\end{array}$ & $\begin{array}{l}\text { Retrospective/skin } \\
\text { examination/excisional biopsy }\end{array}$ \\
\hline \multirow[t]{7}{*}{$\begin{array}{l}\text { Smith et al, } \\
1965\end{array}$} & \multirow[t]{7}{*}{ (14) } & \multirow[t]{7}{*}{7} & 40 & M & Trunk & Ipsilateral axillary & $\begin{array}{l}\text { Retrospective/skin } \\
\text { examination/excisional biopsy }\end{array}$ \\
\hline & & & 45 & M & Leg & $\begin{array}{l}\text { Ipsilateral inguinal } \\
\text { lymph nodes }\end{array}$ & $\begin{array}{l}\text { Retrospective/skin } \\
\text { examination/excisional biopsy }\end{array}$ \\
\hline & & & 29 & $\mathrm{~F}$ & Arm & $\begin{array}{l}\text { Ipsilateral epitrochlear \& } \\
\text { axillary lymph nodes }\end{array}$ & $\begin{array}{l}\text { Retrospective/skin } \\
\text { examination/excisional biopsy }\end{array}$ \\
\hline & & & 27 & M & Arm & $\begin{array}{l}\text { Ipsilateral axillary } \\
\text { lymph nodes }\end{array}$ & $\begin{array}{l}\text { Retrospective/skin } \\
\text { examination/excisional biopsy }\end{array}$ \\
\hline & & & 33 & M & Cervical & $\begin{array}{l}\text { Ipsilateral cervical } \\
\text { lymph nodes }\end{array}$ & $\begin{array}{l}\text { Retrospective/skin } \\
\text { examination/excisional biopsy }\end{array}$ \\
\hline & & & 31 & $\mathrm{~F}$ & Leg & $\begin{array}{l}\text { Ipsilateral inguinal } \\
\text { lymph nodes }\end{array}$ & $\begin{array}{l}\text { Retrospective/skin } \\
\text { examination/excisional biopsy }\end{array}$ \\
\hline & & & 44 & M & Trunk & $\begin{array}{l}\text { Ipsilateral axillary } \\
\text { lymph nodes }\end{array}$ & $\begin{array}{l}\text { Retrospective/skin } \\
\text { examination/excisional biopsy }\end{array}$ \\
\hline $\begin{array}{l}\text { Todd et al, } \\
1966\end{array}$ & (15) & 1 & 31 & M & Cervical & $\begin{array}{l}\text { Ipsilateral cervical } \\
\text { lymph nodes }\end{array}$ & $\begin{array}{l}\text { Retrospective/skin } \\
\text { examination/excisional biopsy }\end{array}$ \\
\hline $\begin{array}{l}\text { Milton et al, } \\
1967\end{array}$ & (16) & 1 & 53 & M & Cervical & $\begin{array}{l}\text { Contralateral cervical } \\
\text { lymph nodes }\end{array}$ & $\begin{array}{l}\text { Retrospective/skin } \\
\text { examination/excisional biopsy }\end{array}$ \\
\hline \multirow[t]{2}{*}{$\begin{array}{l}\text { Cochran et al, } \\
1970\end{array}$} & \multirow[t]{2}{*}{$(17)$} & \multirow[t]{2}{*}{2} & 41 & M & Leg & $\begin{array}{l}\text { Ipsilateral inguinal } \\
\text { lymph nodes }\end{array}$ & $\begin{array}{l}\text { Retrospective/skin } \\
\text { examination/excisional biopsy }\end{array}$ \\
\hline & & & 50 & M & Leg & $\begin{array}{l}\text { Ipsilateral inguinal } \\
\text { lymph nodes }\end{array}$ & $\begin{array}{l}\text { Retrospective/skin } \\
\text { examination/excisional biopsy }\end{array}$ \\
\hline $\begin{array}{l}\text { McGovern, } \\
1975\end{array}$ & (18) & 1 & 57 & M & Trunk & $\begin{array}{l}\text { Supraclavicular and. } \\
\text { cervical lymph nodes }\end{array}$ & $\begin{array}{l}\text { Retrospective/skin } \\
\text { examination/excisional biopsy }\end{array}$ \\
\hline $\begin{array}{l}\text { McDougal et al, } \\
1976\end{array}$ & (19) & 1 & 72 & $\mathrm{~F}$ & Cervical & $\begin{array}{l}\text { Ipsilateral cervical } \\
\text { lymph nodes }\end{array}$ & $\begin{array}{l}\text { Retrospective/skin } \\
\text { examination/excisional biopsy }\end{array}$ \\
\hline $\begin{array}{l}\text { Whicker et al, } \\
1980\end{array}$ & (20) & 1 & 53 & $\mathrm{~F}$ & Cervical & $\begin{array}{l}\text { Ipsilateral cervical } \\
\text { lymph nodes }\end{array}$ & $\begin{array}{l}\text { Retrospective/skin } \\
\text { examination/excisional biopsy }\end{array}$ \\
\hline $\begin{array}{l}\text { Pelligrini, } \\
1980\end{array}$ & (21) & 1 & 50 & $\mathrm{~F}$ & Leg & $\begin{array}{l}\text { Ipsilateral inguinal } \\
\text { lymph nodes }\end{array}$ & $\begin{array}{l}\text { Retrospective/skin } \\
\text { examination/excisional biopsy }\end{array}$ \\
\hline \multirow{2}{*}{$\begin{array}{l}\text { Landthaler and } \\
\text { Braun-Falco, } \\
1981\end{array}$} & \multirow[t]{2}{*}{ (22) } & \multirow[t]{2}{*}{2} & 41 & M & Leg & $\begin{array}{l}\text { Ipsilateral inguinal } \\
\text { lymph nodes }\end{array}$ & $\begin{array}{l}\text { Retrospective/skin } \\
\text { examination/excisional biopsy }\end{array}$ \\
\hline & & & 63 & $\mathrm{~F}$ & Leg & $\begin{array}{l}\text { Ipsilateral inguinal } \\
\text { lymph nodes }\end{array}$ & $\begin{array}{l}\text { Retrospective/skin } \\
\text { examination/excisional biopsy }\end{array}$ \\
\hline $\begin{array}{l}\text { Kessler } \text { et al, } \\
1984\end{array}$ & (23) & 1 & 40 & $\mathrm{~F}$ & Leg & $\begin{array}{l}\text { Ipsilateral inguinal } \\
\text { lymph nodes }\end{array}$ & $\begin{array}{l}\text { Retrospective/skin } \\
\text { examination/excisional biopsy }\end{array}$ \\
\hline $\begin{array}{l}\text { Rampen and } \\
\text { Meijer, } 1985\end{array}$ & (24) & 1 & 37 & M & Cervical & Brain & $\begin{array}{l}\text { Retrospective/skin } \\
\text { examination/excisional biopsy }\end{array}$ \\
\hline $\begin{array}{l}\text { Paul and } \\
\text { Müllhofer, } 1990\end{array}$ & (25) & 1 & 60 & M & Trunk & $\begin{array}{l}\text { Ipsilateral inguinal } \\
\text { lymph nodes }\end{array}$ & $\begin{array}{l}\text { Retrospective/skin } \\
\text { examination/excisional biopsy }\end{array}$ \\
\hline \multirow[t]{2}{*}{$\begin{array}{l}\text { Bottger et al, } \\
1992\end{array}$} & \multirow[t]{2}{*}{ (26) } & \multirow[t]{2}{*}{2} & 41 & M & Leg & $\begin{array}{l}\text { Ipsilateral inguinal } \\
\text { lymph nodes }\end{array}$ & $\begin{array}{l}\text { Retrospective/skin } \\
\text { examination/excisional biopsy }\end{array}$ \\
\hline & & & 63 & $\mathrm{~F}$ & Leg & $\begin{array}{l}\text { Ipsilateral inguinal } \\
\text { lymph nodes }\end{array}$ & $\begin{array}{l}\text { Retrospective/skin } \\
\text { examination/excisional biopsy }\end{array}$ \\
\hline \multirow[t]{6}{*}{$\begin{array}{l}\text { Avril et al, } \\
1992\end{array}$} & \multirow[t]{6}{*}{ (27) } & \multirow[t]{6}{*}{7} & 40 & $\mathrm{~F}$ & Leg & $\begin{array}{l}\text { Ipsilateral inguinal } \\
\text { lymph nodes }\end{array}$ & $\begin{array}{l}\text { Retrospective/skin } \\
\text { examination/excisional biopsy }\end{array}$ \\
\hline & & & 57 & M & Trunk & $\begin{array}{l}\text { Contralateral inguinal } \\
\text { lymph nodes }\end{array}$ & $\begin{array}{l}\text { Retrospective/skin } \\
\text { examination/excisional biopsy }\end{array}$ \\
\hline & & & 42 & M & Trunk & $\begin{array}{l}\text { Ipsilateral axillary } \\
\text { lymph nodes }\end{array}$ & $\begin{array}{l}\text { Retrospective/skin } \\
\text { examination/excisional biopsy }\end{array}$ \\
\hline & & & 68 & M & Arm & $\begin{array}{l}\text { Ipsilateral axillary } \\
\text { lymph nodes }\end{array}$ & $\begin{array}{l}\text { Retrospective/skin } \\
\text { examination/excisional biopsy }\end{array}$ \\
\hline & & & 52 & M & Arm & $\begin{array}{l}\text { Ipsilateral axillary } \\
\text { lymph nodes }\end{array}$ & $\begin{array}{l}\text { Retrospective/skin } \\
\text { examination/excisional biopsy }\end{array}$ \\
\hline & & & 33 & $\mathrm{~F}$ & Trunk & $\begin{array}{l}\text { Ipsilateral axillary } \\
\text { lymph nodes }\end{array}$ & $\begin{array}{l}\text { Retrospective/skin } \\
\text { examination/excisional biopsy }\end{array}$ \\
\hline
\end{tabular}


Table I. Continued.

\begin{tabular}{|c|c|c|c|c|c|c|c|}
\hline Author (year) & Ref. & $\begin{array}{l}\text { No. of } \\
\text { patients }\end{array}$ & $\begin{array}{c}\text { Age } \\
\text { (years) }\end{array}$ & Sex & $\begin{array}{l}\text { Location of } \\
\text { primary tumor }\end{array}$ & Sites of metastasis & $\begin{array}{l}\text { Diagnosis of } \\
\text { primary tumor }\end{array}$ \\
\hline & & & 63 & M & Trunk & $\begin{array}{l}\text { Ipsilateral axillary } \\
\text { lymph nodes }\end{array}$ & $\begin{array}{l}\text { Retrospective/skin } \\
\text { examination/excisional biopsy }\end{array}$ \\
\hline Shai et al, 1994 & (9) & 1 & 53 & $\mathrm{~F}$ & Leg & $\begin{array}{l}\text { Ipsilateral inguinal } \\
\text { lymph nodes }\end{array}$ & $\begin{array}{l}\text { Retrospective/skin } \\
\text { examination/excisional biopsy }\end{array}$ \\
\hline Sais et al, 1994 & (28) & 1 & 57 & $\mathrm{~F}$ & Cervical & $\begin{array}{l}\text { Ipsilateral parotid } \\
\text { gland, brain }\end{array}$ & $\begin{array}{l}\text { Retrospective/skin } \\
\text { examination/excisional biopsy }\end{array}$ \\
\hline \multirow[t]{4}{*}{ High et al, 2005} & (11) & 4 & 68 & $\mathrm{M}$ & Trunk & $\begin{array}{l}\text { Ipsilateral axillary } \\
\text { lymph nodes, brain }\end{array}$ & $\begin{array}{l}\text { Retrospective/skin } \\
\text { examination/excisional biopsy }\end{array}$ \\
\hline & & & 48 & M & Trunk & $\begin{array}{l}\text { Ipsilateral axillary } \\
\text { lymph nodes }\end{array}$ & $\begin{array}{l}\text { Retrospective/skin } \\
\text { examination/excisional biopsy }\end{array}$ \\
\hline & & & 55 & M & Leg & $\begin{array}{l}\text { Ipsilateral inguinal } \\
\text { lymph nodes }\end{array}$ & $\begin{array}{l}\text { Retrospective/skin } \\
\text { examination/excisional biopsy }\end{array}$ \\
\hline & & & 21 & $\mathrm{~F}$ & Leg & $\begin{array}{l}\text { Ipsilateral inguinal } \\
\text { lymph nodes }\end{array}$ & $\begin{array}{l}\text { Retrospective/skin } \\
\text { examination/excisional biopsy }\end{array}$ \\
\hline \multirow[t]{2}{*}{$\begin{array}{l}\text { Emanuel et al, } \\
2008\end{array}$} & (29) & 2 & 40 & $\mathrm{~F}$ & Trunk & $\begin{array}{l}\text { Regional lymph } \\
\text { nodes NOS }\end{array}$ & $\begin{array}{l}\text { skin examination/ } \\
\text { excisional biopsy first }\end{array}$ \\
\hline & & & 53 & $\mathrm{M}$ & Cervical & Soft tissue NOS & $\begin{array}{l}\text { Retrospective/skin } \\
\text { examination/excisional biopsy }\end{array}$ \\
\hline \multirow[t]{7}{*}{$\begin{array}{l}\text { Bories et al, } \\
2008\end{array}$} & (12) & 1 & 63 & M & Trunk & $\begin{array}{l}\text { Bilateral axillary } \\
\text { lymph nodes }\end{array}$ & $\begin{array}{l}\text { Retrospective/skin } \\
\text { examination/Excisional biopsy }\end{array}$ \\
\hline & & 2 & 38 & $\mathrm{~F}$ & Trunk & $\begin{array}{l}\text { Ipsilateral sus-clavicular } \\
\text { lymph nodes }\end{array}$ & $\begin{array}{l}\text { Retrospective/skin } \\
\text { examination/Excisional biopsy }\end{array}$ \\
\hline & & 3 & 48 & M & Foot & $\begin{array}{l}\text { Ipsilateral inguinal } \\
\text { lymph nodes and lung }\end{array}$ & $\begin{array}{l}\text { Retrospective/skin } \\
\text { examination/Excisional biopsy }\end{array}$ \\
\hline & & 4 & 36 & $\mathrm{~F}$ & Cervical & $\begin{array}{l}\text { Ipsilateral cervical } \\
\text { lymph nodes }\end{array}$ & $\begin{array}{l}\text { Retrospective/skin } \\
\text { examination/Excisional biopsy }\end{array}$ \\
\hline & & 5 & 63 & M & Trunk & $\begin{array}{l}\text { Ipsilateral axillary } \\
\text { lymph nodes }\end{array}$ & $\begin{array}{l}\text { Retrospective/skin } \\
\text { examination/Excisional biopsy }\end{array}$ \\
\hline & & 6 & 68 & $\mathrm{~F}$ & Leg & $\begin{array}{l}\text { Ipsilateral inguinal lymph } \\
\text { nodes, iliac nodes and bone }\end{array}$ & $\begin{array}{l}\text { Retrospective/skin } \\
\text { examination/Excisional biopsy }\end{array}$ \\
\hline & & 7 & 78 & $\mathrm{~F}$ & Leg & $\begin{array}{l}\text { Ipsilateral inguinal } \\
\text { lymph nodes }\end{array}$ & $\begin{array}{l}\text { Retrospective/skin } \\
\text { examination/Excisional biopsy }\end{array}$ \\
\hline \multirow[t]{3}{*}{$\begin{array}{l}\text { Margaritescu } \\
\text { et al, } 2014\end{array}$} & $(30)$ & 3 & 45 & $\mathrm{~F}$ & Trunk & $\begin{array}{l}\text { Ipsilateral axillary } \\
\text { lymph nodes }\end{array}$ & $\begin{array}{l}\text { Retrospective/skin } \\
\text { examination/excisional biopsy }\end{array}$ \\
\hline & & & 45 & M & Trunk & $\begin{array}{l}\text { Ipsilateral axillary } \\
\text { lymph nodes }\end{array}$ & skin examination/Biopsy first \\
\hline & & & 40 & M & Trunk & $\begin{array}{l}\text { Brain } \\
\text { Ipsilateral axillary } \\
\text { lymph nodes }\end{array}$ & $\begin{array}{l}\text { Retrospective/skin } \\
\text { examination/excisional biopsy }\end{array}$ \\
\hline $\begin{array}{l}\text { Yamada et al, } \\
2016\end{array}$ & $(31)$ & 1 & 65 & M & Leg & $\begin{array}{l}\text { Ipsilateral inguinal } \\
\text { lymph nodes }\end{array}$ & $\begin{array}{l}\text { Retrospective/skin } \\
\text { examination/excisional biopsy }\end{array}$ \\
\hline \multicolumn{8}{|c|}{ Complete regression of primary tumor after incisional biopsy } \\
\hline $\begin{array}{l}\text { Dunn et al, } \\
2008\end{array}$ & $(32)$ & 1 & 39 & $\mathrm{~F}$ & Trunk & No distant metastasis & $\begin{array}{l}\text { Initial partial biopsy } \\
\text { (SSM } 1.8 \mathrm{~mm} \text { growth } \\
\text { Breslow thickness, vertical } \\
\text { fase) complete regression } \\
\text { in } 12 \text { weeks (excision) }\end{array}$ \\
\hline $\begin{array}{l}\text { Menzies and } \\
\text { McCarthy, } 2015\end{array}$ & (33) & 1 & 62 & M & Leg & & $\begin{array}{l}\text { Initial partial biopsy } \\
\text { (SSM } 0.7 \mathrm{~mm} \text { Breslow } \\
\text { thickness) complete regression } \\
\text { in } 18 \text { months on punch biopsy }\end{array}$ \\
\hline $\begin{array}{l}\text { Khosravi et al, } \\
2016\end{array}$ & (34) & 1 & 49 & M & Trunk & Multiple metastasis & $\begin{array}{l}\text { Initial partial biopsy (ulcerated } \\
\mathrm{NM} 8 \mathrm{~mm} \text { Breslow } \\
\text { thickness) complete regression } \\
\text { in } 4 \text { weeks (excision) }\end{array}$ \\
\hline
\end{tabular}


to differentiate between a completely regressed nevus and a completely regressed melanoma, and they proposed some criteria. In a regressed nevus the epidermis is normal, with preserved rete ridges, no tumor melanosis and a lymphocytic infiltrate that leaves the nevus undisturbed. In completely regressed melanoma the epidermis is rarely normal; it can be atrophic or irregularly hyperplastic. There is dermal fibrosis, sometimes with foci of tumor melanosis and a rich lymphocytic infiltrate. We can also note the presence of an ectatic superficial vasculature (Fig. 1C). A challenging situation is when, like in our case, regression involves a melanoma developed in a nevus (Fig. 1A and B).

Mutational status in completely regressed melanomas. Across human cancer type, melanoma is the tumor with the highest prevalence of somatic mutations (5). Bastian (4) found that BRAF mutational status was highly present in melanoma arising in non-chronic sun damaged skin, but it can be present in a small percentage in melanomas arising on chronic sun damaged skin or glabrous skin. The most common somatic mutation in melanoma is BRAF V600E, present in up to $90 \%$ of BRAF mutated melanomas $(41,42)$. It is associated with younger age at diagnosis and primary lesion located often on the trunk (6). Partial regression, found in 10-35\% of primary melanoma cases, is a common dermoscopic criteria associated with BRAF mutation (39). We present for the first time to our knowledge a case of completely regressed primary melanoma which harbors BRAF V600E mutation in the metastatic lesion and develops a second primary BRAF wild-type melanoma under BRAF inhibitors.

BRAF mutation is present in nevi in a higher percentage than in melanoma $(70-82 \%$ in nevi vs. $50-60 \%$ in melanomas) (43). The introduction of BRAF inhibitor therapy in the treatment of melanoma improved response rates and overall survival in patients with melanoma but with nevi dynamic change as a common side effect. Nevi involution, especially for the papillomatous compound type, increasing in size and pigmentation for the flat lesions but also appearance of new BRAF wild-type melanoma were reported $(36,44)$. This is due to paradoxical activation of MAPK pathway, especially with BRAF inhibitor monotherapy, also associated with resistance to therapy (45).

Spontaneous complete regression - possible mechanisms. A lot of factors leading to complete regression in melanoma were mentioned in the literature. There are exogenous factors like surgical trauma, vaccines (BCG, rabies), UV exposure, medication (BRAF inhibitors) and endogenous factors like pregnancy $(32,33,46-49)$. The host immune system has an important role in tumor regression in melanoma. The occurrence of regression in primary tumor after lymph node or visceral metastases is highly related to an effective immune response against tumor cells $(46,49)$.

In conclusion, we present for the first time to our knowledge a case of completely regressed BRAF V600E mutated melanoma which showed nevi involution and developed a new BRAF wild-type melanoma after therapy with BRAF inhibitors. On one hand, this case favors the role of BRAF mutation in melanoma immune pathogenesis, and on the other hand, shows the importance of clinical and dermoscopic follow-up in melanoma patients. Our review shows that the diagnosis of completely regressed melanoma is very difficult, especially because there are no unifying concepts in assessing complete regression dermoscopically and pathologically.

\section{Acknowledgements}

Not applicable.

\section{Funding}

This manuscript was partially supported by the Romanian Society of Dermato-oncology.

\section{Availability of data and materials}

The datasets used and/or analyzed during the current study are available from the corresponding author on reasonable request.

\section{Authors' contributions}

GLE, AV, LU, RC were responsible for conception of the manuscript, data analysis and contributed to writing the manuscript. NB, CS, SS, EC, OF contributed to data acquisition and the critical revision of the manuscript for important intellectual content. All authors read and approved the final version of manuscript.

\section{Ethics approval and consent to participate}

This study was approved by the Ethics Committee of the 'Iuliu Hatieganu' University of Medicine and Pharmacy (no. 170/12.05.2014; Cluj-Napoca, Romania). All the participants gave their consent to be included in the study.

\section{Patient consent for publication}

The patient gave his written consent for image and data publication.

\section{Competing interests}

Rodica Cosgarea has received speaker fees from Roche Pharma and Novartis Pharma. Grigore Lavinia Elena has received a speaker fee from Novartis Pharma.

\section{References}

1. Mitchell JK and Leslie KS: Melanoma death prevention: Moving away from the sun. J Am Acad Dermatol 68: e169-e175, 2013.

2. Neagu M, Caruntu C, Constantin C, Boda D, Zurac S, Spandidos DA and Tsatsakis AM: Chemically induced skin carcinogenesis: Updates in experimental models (Review). Oncol Rep 35: 2516-2528, 2016.

3. Zurac S, Neagu M, Constantin C, Cioplea M, Nedelcu R, Bastian A, Popp C, Nichita L, Andrei R, Tebeica T, et al: Variations in the expression of TIMP1, TIMP2 and TIMP3 in cutaneous melanoma with regression and their possible function as prognostic predictors. Oncol Lett 11: 3354-3360, 2016.

4. Bastian BC: The molecular pathology of melanoma: An integrated taxonomy of melanocytic neoplasia. Annu Rev Pathol 9: 239-271, 2014. 
5. Alexandrov LB, Nik-Zainal S, Wedge DC, Aparicio SA, Behjati S, Biankin AV, Bignell GR, Bolli N, Borg A, Børresen-Dale AL, et al; Australian Pancreatic Cancer Genome Initiative; ICGC Breast Cancer Consortium; ICGC MMML-Seq Consortium; ICGC PedBrain: Signatures of mutational processes in human cancer. Nature 500: 415-421, 2013.

6. Sullivan RJ (ed): Molecular diagnostics and tumor mutational analysis. In: BRAF Targets in Melanoma-Biological Mechanisms, Resistance and Drug Discovery. Springer, New York, NY, pp47-72, 2015.

7. Carlos G, Anforth R, Clements A, Menzies AM, Carlino MS Chou $S$ and Fernandez-Peñas P: Cutaneous toxic effects of BRAF inhibitors alone and in combination with MEK inhibitors for metastatic melanoma. JAMA Dermatol 151: 1103-1109, 2015

8. Bolognia JL, Schaffer JV and Cerroni L: Neoplasms of the skin. In: Dermatology. 4th Edition. Elsevier, pp1989-2020, 2018.

9. Shai A, Avinoach I and Sagi A: Metastatic malignant melanoma with spontaneous and complete regression of the primary lesion. Case report and review of the literature. J Dermatol Surg Oncol 20: 342-345, 1994

10. Ehrsam E, Kallini JR, Lebas D, Khachemoune A, Modiano P and Cotten H: Fully regressive melanoma: A case without metastasis. J Clin Aesthet Dermatol 9: 42-46, 2016.

11. High WA, Stewart D, Wilbers CR, Cockerell CJ, Hoang MP and Fitzpatrick JE: Completely regressed primary cutaneous malignant melanoma with nodal and/or visceral metastases: A report of 5 cases and assessment of the literature and diagnostic criteria. J Am Acad Dermatol 53: 89-100, 2005.

12. Bories N, Dalle S, Debarbieux S, Balme B, Ronger-Savlé S and Thomas L: Dermoscopy of fully regressive cutaneous melanoma. Br J Dermatol 158: 1224-1229, 2008.

13. Dasgupta T, Bowden L and Berg JW: Malignant melanoma of unknown primary origin. Surg Gynecol Obstet 117: 341-345, 1963

14. Smith JL Jr and Stehlin JS Jr: Spontaneous regression of primary malignant melanomas with regional metastases. Cancer 18 $1399-1415,1965$

15. Todd DW, Farrow GM, Winkelmann RK and Payne WS: Spontaneous regression of primary malignant melanoma with regional metastasis: Report of a case with photographic documentation. Mayo Clin Proc 41: 672-676, 1966.

16. Milton CW, Lane Brown MM and Gilder M: Malignant melanoma with an occult primary lesion. Br J Surg 54: 651-658, 1967.

17. Cochran AJ, Diehl V and Stjernswärd J: Regression of primary malignant melanoma associated with a good prognosis despite metastasis to lymph nodes. Rev Eur Etud Clin Biol 15: 969-972, 1970.

18. McGovern VJ: Spontaneous regression of melanoma. Pathology 7 : 91-99, 1975.

19. MacDougal BA, Weeks PM and Wray RC Jr: Spontaneous regression of the primary lesion of a metastatic malignant melanoma. Plast Reconstr Surg 57: 355-358, 1976.

20. Whicker JH, DeMarco PR and Fitzgibbons JF: Spontaneous regression of a facial malignant melanoma. Arch Otolaryngol 106: $50-51,1980$

21. Pellegrini AE: Regressed primary malignant melanoma with regional metastases. Arch Dermatol 116: 585-586, 1980.

22. Landthaler M and Braun-Falco O: Malignant melanoma with unknown primary tumor. Case reports of 12 patients with overview. Hautarzt 32: 339-344, 1981 (In German).

23. Kessler E, Schwartz P and Antebi E: Spontaneous regression of primary malignant melanoma with metastases. Plast Reconstr Surg 74: 427-429, 1984

24. Rampen FH and Meijer J: Metastatic melanoma of the brain after spontaneous regression of the primary. Acta Neurol Scand 72: 222-224, 1985

25. Paul E and Müllhofer R: Metastasizing malignant melanoma from unknown primary tumor. Hautarzt 41: 432-437, 1990 (In German).

26. Bottger D, Dowden RV and Kay PP: Complete spontaneous regression of cutaneous primary malignant melanoma. Plast Reconstr Surg 89: 548-553, 1992.

27. Avril MF, Charpentier P, Margulis A and Guillaume JC: Regression of primary melanoma with metastases. Cancer 69: 1377-1381, 1992.

28. Sais G, Marcoval J, Juggla A, Curco N and Servitje O Dermatomyositis and metastatic malignant melanoma, with complete regression of the primary lesion. Br J Dermatol 130: 796-797, 1994

29. Emanuel PO, Mannion M and Phelps RG: Complete regression of primary malignant melanoma. Am J Dermatopathol 30: 178-181, 2008.
30. Mărgăritescu I, Chiriţă AD and Vasilescu F: Completely regressed primary cutaneous melanoma - difficulties in diagnosis and classification. Rom J Morphol Embryol 55 (Suppl 2): 635-642, 2014.

31. Yamada S, Nawata A, Yoshioka M, Hiraki T, Higashi M, Hatanaka $\mathrm{K}$ and Tanimoto A: Complete regression of primary cutaneous malignant melanoma associated with distant lymph node metastasis: A teaching case mimicking blue nevus. BMC Res Notes 9: 366, 2016

32. Dunn GP, Lewis JS Jr, Sunwoo JB and Uppaluri R: Spontaneous regression of cutaneous head and neck melanoma: Implications for the immunologic control of neoplasia. Head Neck 30: 267-272, 2008.

33. Menzies SW and McCarthy WH: Complete regression of primary cutaneous malignant melanoma. Arch Surg 132: 553-556, 1997.

34. Khosravi H, Akabane AL, Alloo A, Nazarian RM and Boland GM: Metastatic melanoma with spontaneous complete regression of a thick primary lesion. JAAD Case Rep 2: 439-441, 2016.

35. Martín JM, Pinazo I, Monteagudo C, Markovic J, Allende A and Jordá E: Spontaneous regression of multiple melanocytic nevi after melanoma: Report of 3 cases. Am J Dermatopathol 36: e183-e188, 2014.

36. McClenahan P, Lin LL, Tan JM, Flewell-Smith R, Schaider H, Jagirdar K, Atkinson V, Lambie D, Prow TW, Sturm RA, et al: BRAFV600E mutation status of involuting and stable nevi in dabrafenib therapy with or without trametinib. JAMA Dermatol 150: 1079-1082, 2014.

37. Argenziano G, Zalaudek I, Corona R, Sera F, Cicale L, Petrillo G, Ruocco E, Hofmann-Wellenhof R and Soyer HP Vascular structures in skin tumors: A dermoscopy study. Arch Dermatol 140: 1485-1489, 2004.

38. Zalaudek I, Argenziano G, Ferrara G, Soyer HP, Corona R, Sera F, Cerroni L, Carbone A, Chiominto A, Cicale L, et al: Clinically equivocal melanocytic skin lesions with features of regression: A dermoscopic-pathological study. Br J Dermatol 150: 64-71, 2004.

39. Pozzobon FC, Puig-Butillé JA, González-Alvarez T, Carrera C, Aguilera P, Alos L, Badenas C, Grichnik JM, Malvehy J and Puig S: Dermoscopic criteria associated with BRAF and NRAS mutation status in primary cutaneous melanoma. Br J Dermatol 171: 754-759, 2014

40. Massi G and LeBoit PE: Histological Diagnosis of Nevi and Melanoma. Springer, Berlin, Heidelberg, pp385-398, 2014.

41. Cheng L, Lopez-Beltran A, Massari F, MacLennan GT and Montironi R: Molecular testing for BRAF mutations to inform melanoma treatment decisions: A move toward precision medicine. Mod Pathol 31: 24-38, 2018.

42. Diaconeasa A, Boda D, Solovan C, Enescu DM, Vîlcea AM and Zurac S: Histopathologic features of Spitzoid lesions in different age groups. Rom J Morphol Embryol 54: 51-62, 2013.

43. Pollock PM, Harper UL, Hansen KS, Yudt LM, Stark M, Robbins CM, Moses TY, Hostetter G, Wagner U, Kakareka J, et al: High frequency of BRAF mutations in nevi. Nat Genet 33: 19-20, 2003.

44. Haenssle HA, Kraus SL, Brehmer F, Kretschmer L, Völker B, Asper H, Kapp A and Gutzmer R: Dynamic changes in nevi of a patient with melanoma treated with vemurafenib: Importance of sequential dermoscopy. Arch Dermatol 148: 1183-1185, 2012.

45. Weeraratna AT: RAF around the edges - the paradox of BRAF inhibitors. N Engl J Med 366: 271-273, 2012.

46. Aung PP, Nagarajan P and Prieto VG: Regression in primary cutaneous melanoma: Etiopathogenesis and clinical significance. Lab Invest 97: 657-668, 2017.

47. Lupu M, Caruntu A, Caruntu C, Papagheorghe LML, Ilie MA, Voiculescu V, Boda D, Constantin C, Tanase C, Sifaki M, et al: Neuroendocrine factors: The missing link in non-melanoma skin cancer (Review). Oncol Rep 38: 1327-1340, 2017.

48. Caruntu C, Boda D, Constantin C, Caruntu A and Neagu M: Catecholamines increase in vitro proliferation of murine B16F10 melanoma cells. Acta Endocrinol (Copenh) 10: 545-558, 2014.

49. Boda D: Cellomics as integrative omics for cancer. Curr Proteomics 10: 237-245, 2013.

This work is licensed under a Creative Commons Attribution-NonCommercial-NoDerivatives 4.0 International (CC BY-NC-ND 4.0) License. 